\title{
ESTUDO DA DISSOLUÇÃO DA ALUMINA NO BANHO ELETROLÍTICO NA PRODUÇÃO DE ALUMÍNIO PRIMÁRIO EM CUBAS DE TECNOLOGIA VSS SODERBERG*
}

Diego Cota Marinho

Marcelo Breda Mourão

\begin{abstract}
Resumo
O processo de dissolução da alumina em condições industriais em cubas de Tecnologia VSS Soderberg de alimentações laterais em batelada é bastante complexo, pois sofre interferência de muitas variáveis de processo como: temperatura e química de banho, propriedades físico-químicas da alumina, quantidade de alumina adicionada e massa de banho disponível. Um bom controle de adição e variáveis de processo contribuirão para garantia de uma melhor eficiência de corrente, consumo de energia e redução da quantidade lama (alumina não dissolvida) formada que se deposita no fundo das cubas e irá comprometer a passagem da corrente elétrica e consequentemente reduzirá a eficiência dos fornos. Este estudo mostra as principais variáveis que influenciam no processo de dissolução, o comportamento da concentração da alumina e temperatura do banho durante os intervalos de alimentação de 2:40 e 4:00 horas, a temperatura de entrada da alumina e as condições de cavidade devido a deposição de lama no fundo das cubas.
\end{abstract}

Palavras-chave: Dissolução da alumina; Lama; Temperatura de banho.

\section{STUDY OF ALUMINA DISSOLUTION IN CRYOLITIC BATH TO THE VERTICAL SODERBERG (VSS) ALUMINIUM PRODUCTION PROCESS}

\section{Abstract}

The dissolution process of alumina on industrial conditions in side feeding VSS Soderberg technology is very complex, because suffers interference from process variables such as: temperature and bath chemistry, physicochemical properties of alumina, amount of alumina added and bath mass available. A good control of alumina addition and process variables will contribute to keep good the current efficiency, energy consumption and reduction of the amount of sludge (undissolved alumina) formed at the bottom that in the future will compromise the flow of the electric current that will consequently reduce the efficiency of the pots. This study will show the main variables that influence the dissolution process, the behavior of the alumina concentration and the bath temperature during the feeding intervals of 2:40 and 4:00 hours, the inlet temperature of the alumina and the cavity conditions due the deposition of sludge on the bottom.

Keywords: Dissolution of alumina; sludge formation; Bath Temperature.

1 Engenheiro Metalurgista, Coordenador de Operações, Sala Fornos, Votorantim CBA, Alumínio, São Paulo, Brasil.

2 Membro da ABM, Engenheiro Metalurgista, Professor Livre Docente do Departamento de Engenharia Metalúrgica e de Materiais da Escola Politécnica da Universidade de São Paulo, São Paulo, Brasil. 


\section{INTRODUÇÃO}

A produção do alumínio primário através do processo Hall Heroult, acontece pela passagem da corrente elétrica pelo banho eletrolítico (aproximadamente $80 \%$ de criolita, $\mathrm{Na}_{3} \mathrm{AlF}_{6}, 10 \%$ de fluoreto de alumínio $\mathrm{AlF}_{3}, 5 \%$ de fluorita $\left(\mathrm{CaF}_{2}\right)$ ) que reduz a alumina $\left(\mathrm{Al}_{2} \mathrm{O}_{3}\right)$ disponível no banho (equação 1 ). A medida que a alumina é consumida a concentração da mesma no banho diminui se fazendo necessárias adições desta matéria prima. Nos processos de tecnologia VSS Soderberg de alimentação lateral, a adição ocorre em batelada em intervalos de tempo prédefinidos. Na Votorantim CBA estudou-se a adição em intervalos de 2:40 e 4:00 horas.

$2 \mathrm{Al}_{2} \mathrm{O}_{3}(\mathrm{~s})+3 \mathrm{C}_{(\mathrm{s})} \rightarrow 4 \mathrm{Al}(\mathrm{l})+3 \mathrm{CO}_{2}(\mathrm{~g})$

O processo de dissolução da alumina no banho é bastante complexo (equação 2), para ocorrer ela precisa passar pelas etapas de pré-aquecimento, sair da temperatura da crosta e atingir a temperatura de dissolução (temperatura do eletrólito, aproximadamente $960^{\circ} \mathrm{C}$ ), transferência de calor do eletrólito para ela e também pelo processo endotérmico de dissolução da alumina [1]. Portanto sua velocidade é influenciada por muitas variáveis, onde as principais são: Temperatura (tanto do banho, quanto da entrada da $\mathrm{Al}_{2} \mathrm{O}_{3}$ ), composição química do banho, massa de banho, propriedades físico-químicas e quantidade de alumina adicionada. No caso da Votorantim CBA que trabalha em regime de adições em batelada onde adiciona-se alumina a aproximadamente $550^{\circ} \mathrm{C}$ em quantidades superiores a $180 \mathrm{~kg}$ em uma massa de banho de 5 toneladas a uma temperatura de banho de $960^{\circ} \mathrm{C}$ este processo torna-se ainda mais complexo, pois também passam por um processo de aglomeração (encapsulamento) da alumina adicionada que necessita de uma maior demanda energética para se dissolver.

$2 \mathrm{Al}_{2} \mathrm{O}_{3(\mathrm{~s})} \rightarrow 4 \mathrm{Al}^{+3}(\mathrm{~d})+3 \mathrm{O}_{2}(\mathrm{~g})$

Cada variável influencia no processo de maneira diferente e muitas vezes "prejudica" uma propriedade e melhora outras; a seguir as principais variáveis e como elas influenciam na dissolução e processo.

\subsection{Temperatura de Operação e Superheat $(\Delta T)$}

A temperatura do banho é função da composição do eletrólito e do balanço térmico. Sendo um dos fatores mais importantes no processo de obtenção do alumínio, seu controle é fundamental para garantir a eficiência de corrente, solubilidade da alumina no banho, consumos de energia e pasta anódica [2].

Tradicionalmente a temperatura de operação de uma célula é representada por:

$T=T_{\text {fusão }}+\Delta \mathrm{T}$, sendo $\Delta \mathrm{T}>0$

Onde $T_{\text {fusão }}$ é a temperatura de fusão do eletrólito e $\Delta T$ a temperatura acima do ponto de fusão na qual o eletrólito trabalha [3]. $\mathrm{O} \Delta \mathrm{T}$, também chamado de superheat é dependente da entrada de energia no sistema e do desenho da célula. Normalmente, fornos com alimentação lateral operam com um $\Delta T$ em torno de $10 \mathrm{a}$ $15^{\circ} \mathrm{C}[4]$.

Em processos industriais, buscam-se aditivos que reduzem a temperatura de fusão do eletrólito, o que é considerado vantajoso desde que se possa abaixar a temperatura de operação e consequentemente aumentar a eficiência de corrente [2; 5] do processo devido a menor solubilidade e difusividade do metal no banho, aumento também da tensão interfacial e viscosidade do banho, contribuindo para reduzir a taxa de reoxidação do metal [6; 7]. 
Baixas temperaturas poderão acarretar em um excesso de lombo (material solidificado formado principalmente por criolita sobre as paredes laterais da cuba devido a troca de calor com o sistema) e formação de lama, deteriorando a distribuição de corrente catódica, podendo a eficiência de corrente [3], por esta razão, decrescer mais que o aumento obtido pelo abaixamento da temperatura. Pela sensibilidade enfrentada pelo processo é de extrema importância o bom controle da temperatura ao longo do tempo. Assim se fazem necessários a garantia de um bom aquecimento da alumina antes da entrada na cuba e também na quantidade de alumina adicionada. Segundo [1] a energia requerida no processo de dissolução da alumina varia em uma entalpia em torno de $\Delta$ Hdissolução $=106$ a $130 \mathrm{~kJ} / \mathrm{mol} \mathrm{Al}_{2} \mathrm{O}_{3} \mathrm{e}$ entre 90 a $112 \mathrm{~kJ} / \mathrm{mol}_{\mathrm{Al}_{2} \mathrm{O}_{3}}$ para o processo de aquecimento da alumina até a temperatura de dissolução. A soma das energias requeridas no processo pode reduzir a temperatura do eletrólito em até $15^{\circ} \mathrm{C}$ dependendo da quantidade adicionada e temperatura inicial da alumina. Por este motivo é muito importante um bom controle de adição de alumina no banho.

\subsection{Banho Eletrolítico}

O banho eletrolítico é um sal fundido composto majoritariamente por criolita $\left(\mathrm{Na}_{3} \mathrm{AlF}_{6}\right)$, produto de uma solução de fluoreto de alumínio $\left(\mathrm{AlF}_{3}\right)$ e fluoreto de sódio (NaF), por isso também é conhecido como banho criolítico, cujo ponto de fusão é $1012^{\circ} \mathrm{C}$ [8] e tem a função de prover as melhores condições para as reações ocorrerem. Esse banho pode ser enriquecido com a adição de outros compostos como: fluoreto de cálcio $\left(\mathrm{CaF}_{2}\right)$; fluoreto de manganês $\left(\mathrm{MgF}_{2}\right)$; fluoreto de lítio $(\mathrm{LiF})$; cloreto de sódio $(\mathrm{NaCl})$ e fluoreto de alumínio $\left(\mathrm{AlF}_{3}\right)$. Sendo os aditivos mais utilizados nas indústrias de alumínio o fluoreto de alumínio e fluoreto de cálcio, também chamado de Fluorita [6; 9].

Cada aditivo confere ao banho eletrolítico mudanças em determinadas propriedades, onde a principal característica é a redução do ponto de fusão do eletrólito [10;11]. Uma vez definida a química do banho é necessário que se busque a minimização das variações dos valores de composição química, pois um alto desvio padrão entre os valores de composição química no tempo significa perda de eficiência operacional [10; 11].

Um componente de destaque na química de banho é o fluoreto de alumínio (acidez), que é controlado de forma sistemática pelas equipes de controle de processo. Este aditivo tem como característica principal a redução da temperatura de fusão (Liquidus), redução da solubilidade do alumínio no banho, aumento da tensão interfacial, da viscosidade e da densidade do eletrólito. Os principais efeitos indesejados são a redução da solubilidade da alumina e aumento da resistividade elétrica do banho [2; 6; 9; 10].

\subsection{Propriedades da Alumina}

As propriedades mais relevantes da alumina são: Superfície específica (SSA), perdas por ignição (lost on ignition-LOI), formação cristalográfica, distribuição granulométrica, índice de atrito e densidade. Abaixo as propriedades que mais influenciam no processo de dissolução da alumina no banho.

\subsubsection{Superfície Específica}

A superfície específica das partículas de alumina, também conhecida como SSA, nada mais é do que sua área superficial dividida por unidade de massa. A SSA é definida na síntese da alumina e definida por outras propriedades como tamanho 
das partículas, formação cristalográfica e morfologia dos grãos. Para uso em células de redução a literatura sugere como especificação um valor entre 20 e 100 m²/g.

Quanto maior a superfície específica das partículas maior será a taxa de dissolução, devido à tensão interfacial alumina-banho, as partículas adicionadas não afundam imediatamente havendo tempo suficiente para que cada partícula seja "molhada" pelo banho. Assim, mais área superficial significa maior tensão interfacial permitindo a formação de uma camada de alumina na interface metal-banho sem que as partículas de alumina afundem [12]. Esse efeito também melhora as condições de troca de calor e massa promovendo a dissolução.

\subsubsection{Formação Cristalográfica}

Estudos revelam a existência de pelo menos sete fases cristalográficas para as aluminas calcinadas, livres de água: fases alfa, gama, delta, eta, teta, kappa e chi. A quantidade de alfa-alumina $(\alpha)$ e gama-alumina $(\delta)$ é definida pelas condições de calcinação; calcinações mais severas tendem a formar mais alfa-alumina [13]. A fase mais comum e termodinamicamente estável é a ( $\alpha)$ e a estrutura de transição mais trabalhada é a $(\delta)$, material mais reativo que a alfa-alumina [14], portanto a alumina $(\delta)$ tem maior taxa de dissolução que a alumina a [13; 15]. Esta diferença se deve basicamente às diferentes entalpias de dissolução à temperatura do banho: 27,5 para $\alpha$ e $26,2 \mathrm{Kcal} / \mathrm{mol}$ para $\delta$ [11].

Classificada de acordo com a quantidade de suas fases a alumina Sandy possui teor mais alto de gama-alumina, percentual Alfa (a) inferior a $30 \%$ sendo por esta razão utilizada no processo de redução [11].

\section{MATERIAIS E MÉTODOS}

Foram estudados 2 tipos de alimentação em batelada, 2:40 e 4:00 horas em 3 cubas diferentes. Os principais parâmetros de processo estão mostrados na tabela 1 abaixo:

Tabela 1 - Principais parâmetros de Processo da CBA

\begin{tabular}{|l|l|c|c|c|}
\hline Cuba & Unidade & $\mathbf{7 3 9}$ & $\mathbf{1 6 3 2}$ & $\mathbf{2 0 1 5}$ \\
\hline $\begin{array}{l}\text { Intervaldo de } \\
\text { alimentação }\end{array}$ & horas & $2: 40 \times 2: 40$ & $4: 00 \times 4: 00$ & $2: 40 \times 2: 40$ \\
\hline Quantidade alimentada & $\mathrm{kg}$ & 180 & 300 & 190 \\
\hline Corrente de operação & $\mathrm{kA}$ & 128,0 & 129,5 & 129,0 \\
\hline \%AlF $_{\mathbf{3}}$ (alvo) & $\%$ & 9,3 & 9,3 & 9,3 \\
\hline \%CaF $_{2}$ (alvo) & $\%$ & 5,5 & 5,5 & 5,5 \\
\hline Temperatura (alvo) & ${ }^{\circ} \mathrm{C}$ & 958 & 958 & 958 \\
\hline Produção & $\mathrm{kg} / \mathrm{dia}$ & 920 & 930 & 930 \\
\hline
\end{tabular}

Para entender o comportamento da concentração da alumina no tempo foram coletadas amostras de cubas em condições normais de operação e intervalos de tempo pré-definidos mostrados na tabela 2. As amostras coletadas diretamente dos fornos, após resfriamento foram analisadas quanto ao teor de oxigênio contido utilizando equipamento LECO. A partir do teor de oxigênio estima-se o percentual de alumina. $O$ padrão utilizado para a calibração e verificação do equipamento tem o teor certificado de oxigênio de $0,305 \%$ mais ou menos $0,006 \%$, sendo que o padrão tem massa de aproximadamente $0,1 \mathrm{~g}$. Para a realização dos ensaios foi feita apenas separação em pequenos pedaços e o método foi adaptado utilizando uma 
massa de 0,02g das amostras, para que as mesmas ficassem dentro da curva de calibração com um resultado confiável de até 3\% de oxigênio.

Tabela 2 - Número de amostras por intervalo de tempo

\begin{tabular}{|c|c|c|}
\hline & \multicolumn{2}{|c|}{ Intervalo de Quebra } \\
\hline Condição & $\mathbf{0 2 : 4 0}$ & $\mathbf{0 4 : 0 0}$ \\
\hline Pré-quebra & 2 & 1 \\
\hline 15min após & 3 & 1 \\
\hline 30min após & 3 & 1 \\
\hline 60min após & 3 & 1 \\
\hline 120min após & 2 & 1 \\
\hline 150min após & 2 & 1 \\
\hline E.A & 1 & 0 \\
\hline
\end{tabular}

As temperaturas foram coletadas pela equipe especializada de processo da CBA através de um furo na crosta onde se faz a imersão de um termopar do tipo K no banho a uma profundidade de mais ou menos $9 \mathrm{~cm}$ até a estabilização da temperatura pelo termômetro que está acoplado no termopar. Para se ter maior confiabilidade e entender o comportamento da temperatura entre os ciclos, foram medidas 11 cubas para alimentação de 4 em 4 horas e 22 cubas para alimentação de 2:40 e 2:40 horas nos intervalos de tempo de acordo com a tabela 3.

Tabela 3 - Quantidade de amostras de Temperatura por intervalo de tempo

\begin{tabular}{|c|c|c|}
\hline & \multicolumn{2}{|c|}{ Intervalo de quebra } \\
\hline Condição & $\mathbf{0 2 : 4 0}$ & $\mathbf{0 4 : 0 0}$ \\
\hline Pré-quebra & 22 & 11 \\
\hline Quebra & 22 & 11 \\
\hline $15 \mathrm{~min}$ após & 22 & 11 \\
\hline $30 \mathrm{~min}$ após & 22 & 11 \\
\hline $60 \mathrm{~min}$ após & 22 & 11 \\
\hline 90min após & 22 & 11 \\
\hline $120 \mathrm{~min}$ após & 22 & 11 \\
\hline $150 \mathrm{~min}$ após & 22 & 11 \\
\hline $180 \mathrm{~min}$ após & & 11 \\
\hline $210 \mathrm{~min}$ após & & 11 \\
\hline $240 \mathrm{~min}$ após & & 11 \\
\hline
\end{tabular}

\section{RESULTADOS E DISCUSSÃO}


Antes de iniciar os comentários e análises dos resultados é importante destacar que os testes foram feitos em ambiente industrial (nas linhas de reduções eletrolíticas da CBA) onde consequentemente ocorrem maiores variações quando comparadas a teoria e testes realizados em laboratórios, devido a interferências de outras variáveis inerentes ao processo.

\subsection{Concentração no tempo}

Após o recebimento das análises, estimou-se a concentração de alumina no banho considerando que todo oxigênio contido na amostra correspondia a molécula de alumina, assim obtendo os seguintes resultados (figura 1).

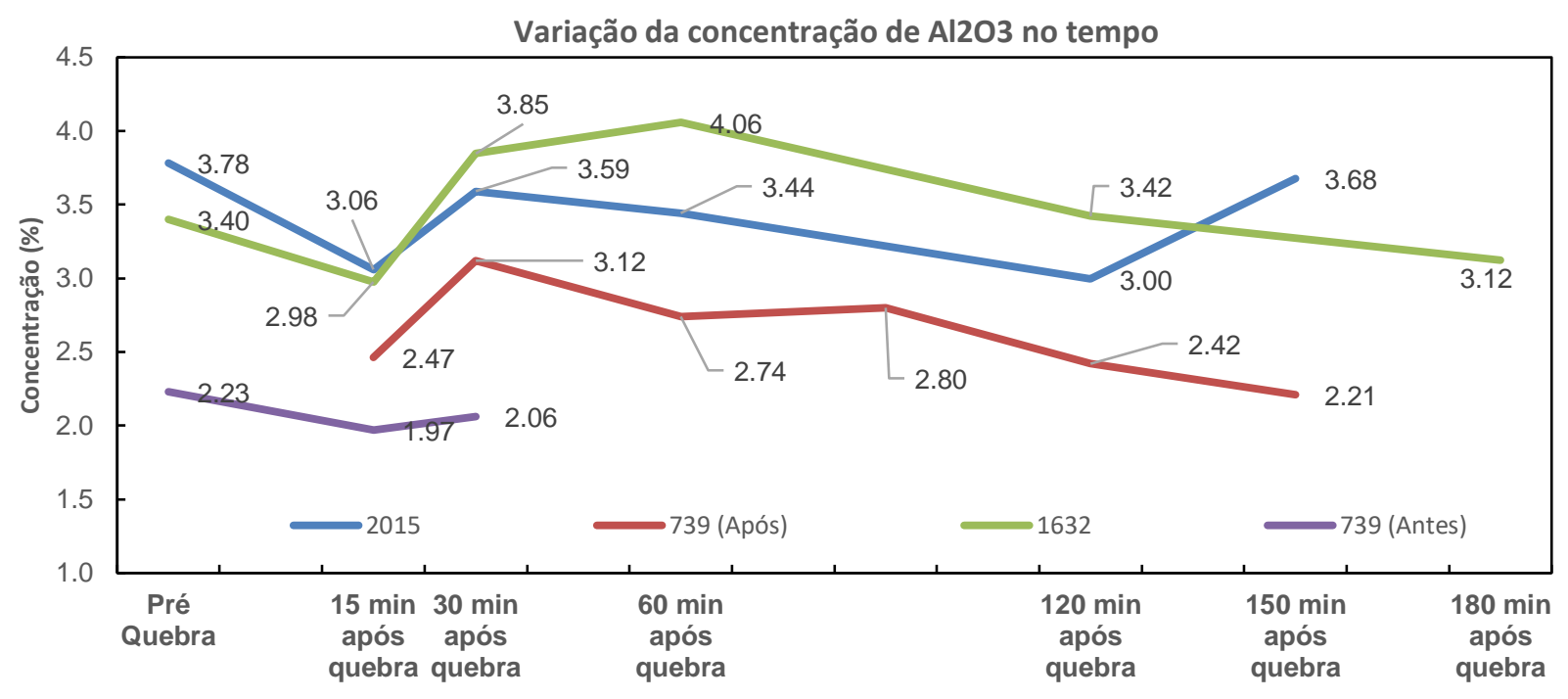

Figura 1 - Variação da concentração de alumina no tempo para os intervalos de alimentação de 2:40 e 4:00 horas.

Avaliando as curvas de concentração, percebe-se que nos 3 casos, imediatamente após a alimentação (quebra), as concentrações de alumina no eletrólito se reduzem, fato que foi associado ao local da amostragem (lado oposto a alimentação), consequentemente é necessário um tempo para homogeneização da concentração do banho.

Fazendo uma análise por tipo de alimentação, percebeu-se comportamento semelhante em ambos os casos, onde se tem uma redução da concentração de alumina nos primeiros 15 minutos após alimentação e em sequência é constatado uma elevação da concentração. No caso de 2:40 horas, esta elevação ocorre até 30 minutos, e para 4:00 horas até 60 minutos. Após esta subida a concentração decresce ao longo do tempo até a próxima alimentação. A diferença entre os tempos de elevação da concentração e redução dos mesmos para os dois tipos diferentes de alimentação foi atribuída a quantidade de alumina adicionada por tipo de alimentação, onde utilizou-se aproximadamente, $180 \mathrm{~kg}$ para 2:40 horas na cuba $739,300 \mathrm{~kg}$ para 4:00 horas na cuba 1632 e $190 \mathrm{~kg}$ para 2:40 na cuba 2015. Mesmo com as balanças dos carros alimentares calibradas com erro de $5 \%$, não se consegue saber a quantidade real de alumina que entra no forno por alimentação.

Para a cuba 739 foi retirada a observação do efeito anódico (E.A) e dividiu-se a curva de concentração em 2 momentos distintos, antes 739 (antes) e depois 739(após) do efeito anódico, fenômeno que ocorre quando a concentração de alumina no banho se reduz para teores inferiores a $2 \%$, fato este que não foi 
explorado no presente trabalho. Mesmo assim o comportamento da concentração no tempo é semelhante as demais.

\subsection{Comportamento da temperatura}

De acordo com a Figura 2, a temperatura de operação durante o regime de alimentação das cubas varia ao longo do tempo, diminuindo após a quebra e atingindo valores mais elevados instantes antes da próxima alimentação. Esta queda ocorre devido a 2 fatores principais: troca de calor da alumina a uma temperatura média de aproximadamente $550^{\circ} \mathrm{C}$ e o banho a $960^{\circ} \mathrm{C}$ e também a reação endotérmica de dissolução da alumina. A subida da temperatura é devida a elevação da temperatura da alumina até a temperatura de dissolução e a eletrólise, tendo como consequência a redução da taxa de troca de calor alumina/banho e também a vedação da cuba pela nova crosta formada, reduzindo assim as perdas de calor para o ambiente externo.

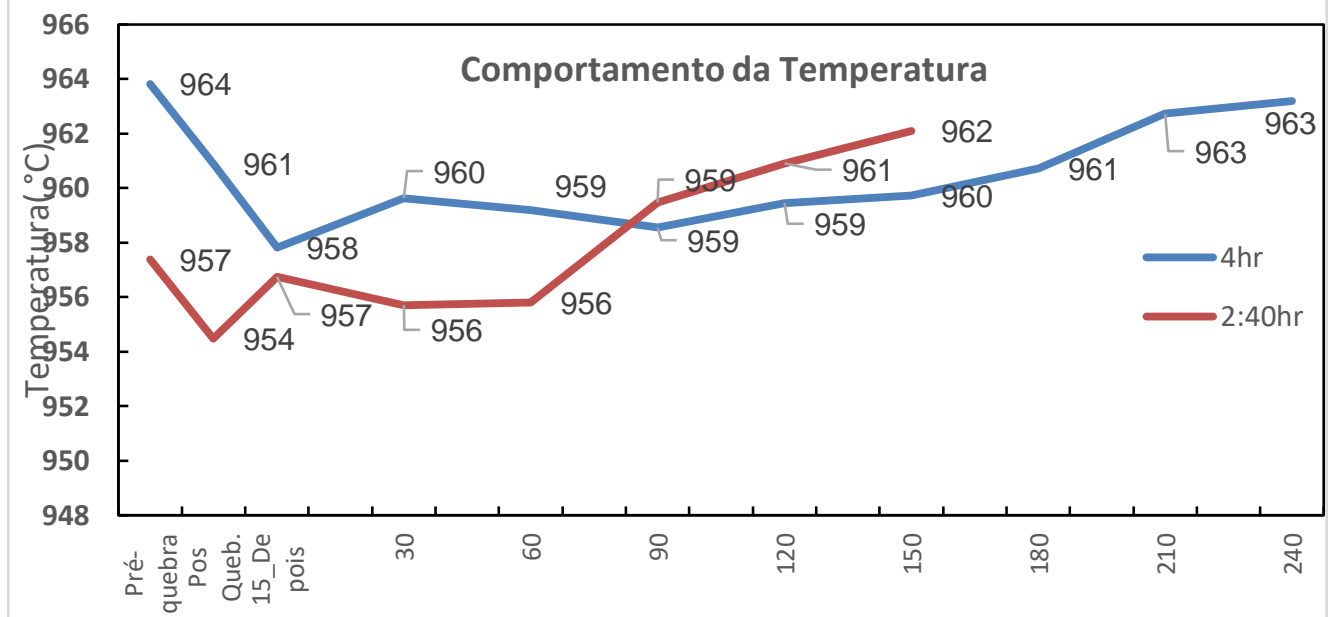

Figura 2 - Variação da temperatura no tempo para os intervalos de alimentação de 2:40 e 4:00 horas.

Para comparar a temperatura nos diferentes regimes de alimentação, com a utilização do MINITAB plotou-se a figura 3 , que mostra uma diferença estatística entre os dois regimes de alimentação comprovada através do teste de hipóteses.

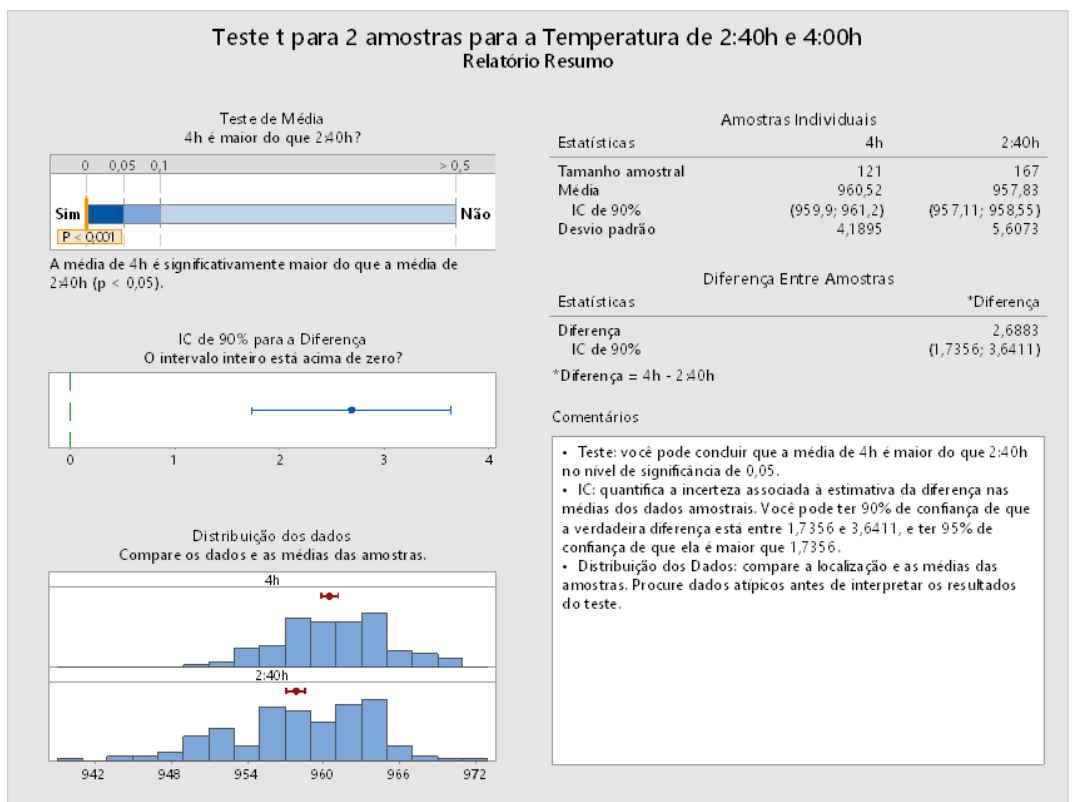

Figura 3 - Teste de Hipótese comparando a temperatura para a alimentação de 2:40 e 4:00 horas. 
O teste de hipótese apresentado pela figura 3 foi realizado através dos dados de temperatura no tempo de intervalo de alimentação para uma amostragem de 11 cubas com intervalo de quebra de 4:00 horas e 22 cubas com intervalo de quebra de $2: 40$ horas. E como resultado, mostrou-se com um intervalo de confiança de $90 \%$ que a diferença entre as temperaturas opera no intervalo entre 1,74 e $3,64^{\circ} \mathrm{C}$. E que estatisticamente podemos afirmar que a média da temperatura durante a quebra de 4:00 horas é $2,69^{\circ} \mathrm{C}$ acima da temperatura de $2: 40$ horas.

Apesar de poder afirmar que as temperaturas são diferentes, esta diferença na temperatura geral dos ciclos não se mostrou muito elevada quando comparamos os dois diferentes regimes de alimentação (2:40h e 4:00h).

Sabe-se que em temperaturas baixas é favorecida a formação da lama e cascão [1] e para contribuir na compreensão da possibilidade do processo de formação da lama em ambos regimes de alimentação, de maneira semelhante à figura 3 , utilizouse o MINITAB com o teste de hipótese para comparar as temperaturas dos 2 regimes de quebra para os tempos de15 minutos e 30 minutos após a quebra (intervalos de tempo onde foram registradas as menores temperaturas durante 0 regime), nas figuras 4 e 5 respectivamente.

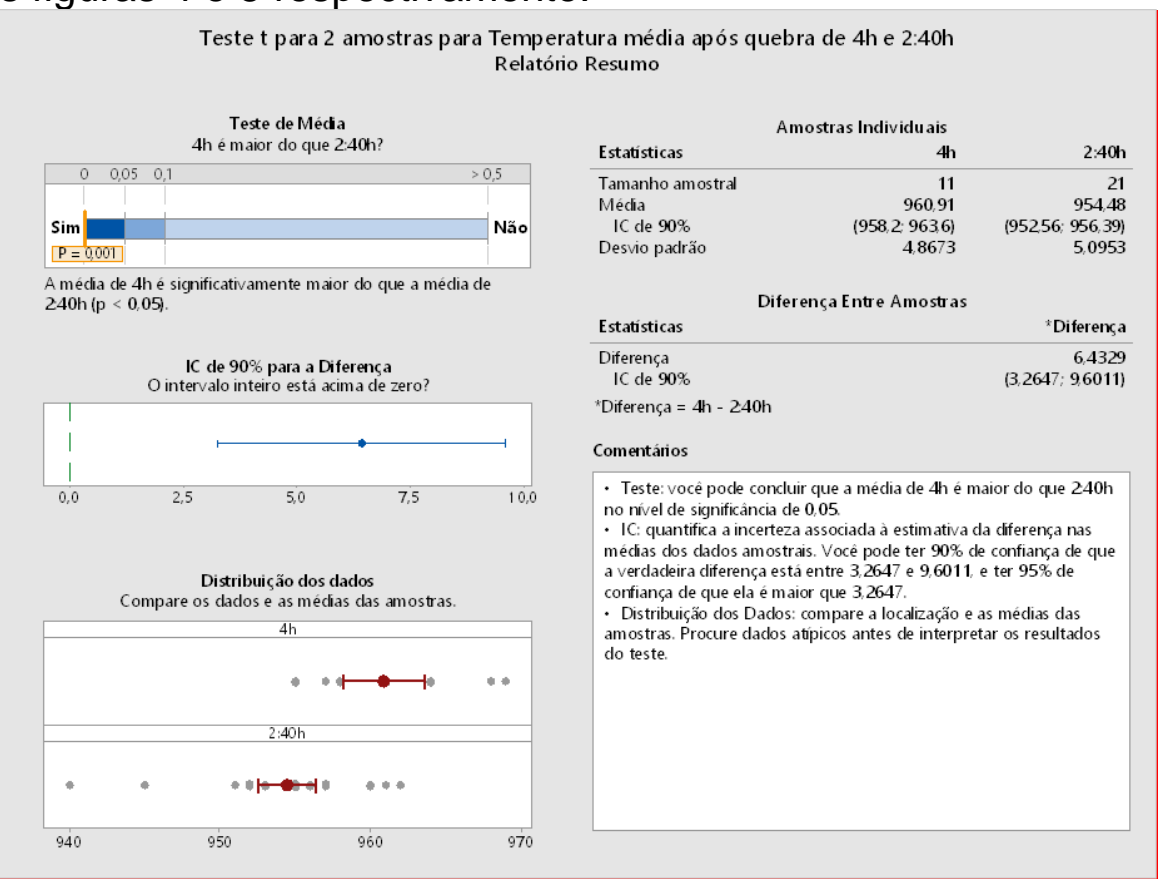

Figura 4 - Teste de Hipótese comparando a temperatura 15 minutos após a quebra para os intervalos de 2:40 e 4:00 horas.

A figura 4 foi construída utilizando a temperatura 15 minutos após quebra e as diferenças obtidas encontradas foram ainda mais significativas do que as da comparação da média de temperatura geral. Como resultado mostrou-se com um intervalo de confiança de $90 \%$ que a diferença entre as temperaturas operam no intervalo entre 3,3 e $9,6^{\circ} \mathrm{C}$ e com uma média para o intervalo de quebra de 4:00 horas, $6,4^{\circ} \mathrm{C}$ acima da temperatura de 2:40 horas. Ao se fazer uma avaliação mais detalhada da temperatura neste ponto específico, percebe-se que apesar de ambos os casos apresentarem um desvio padrão de temperatura bem semelhantes $(4,9$ e $5,1)$ as temperaturas para intervalo de $2: 40$ atingem valores significativamente baixos, estimados de acordo com a amostragem entre 952,6 e $956,4^{\circ} \mathrm{C}$, e alguns pontos com amostras chegando abaixo de $950^{\circ} \mathrm{C}$. ou seja, em muitos casos reduzse a temperatura em níveis que favorecem a formação de lama e cascão nos fornos. Comparando com a quebra de 4:00 horas em que as temperaturas se mostraram 
entre 958,2 a $963,6^{\circ} \mathrm{C}$, com uma média de $960,9^{\circ} \mathrm{C}$, não apresentando amostras com temperaturas baixas e por operarem em uma temperatura superior, favorecem em menor intensidade o processo de formação da lama e cascão.

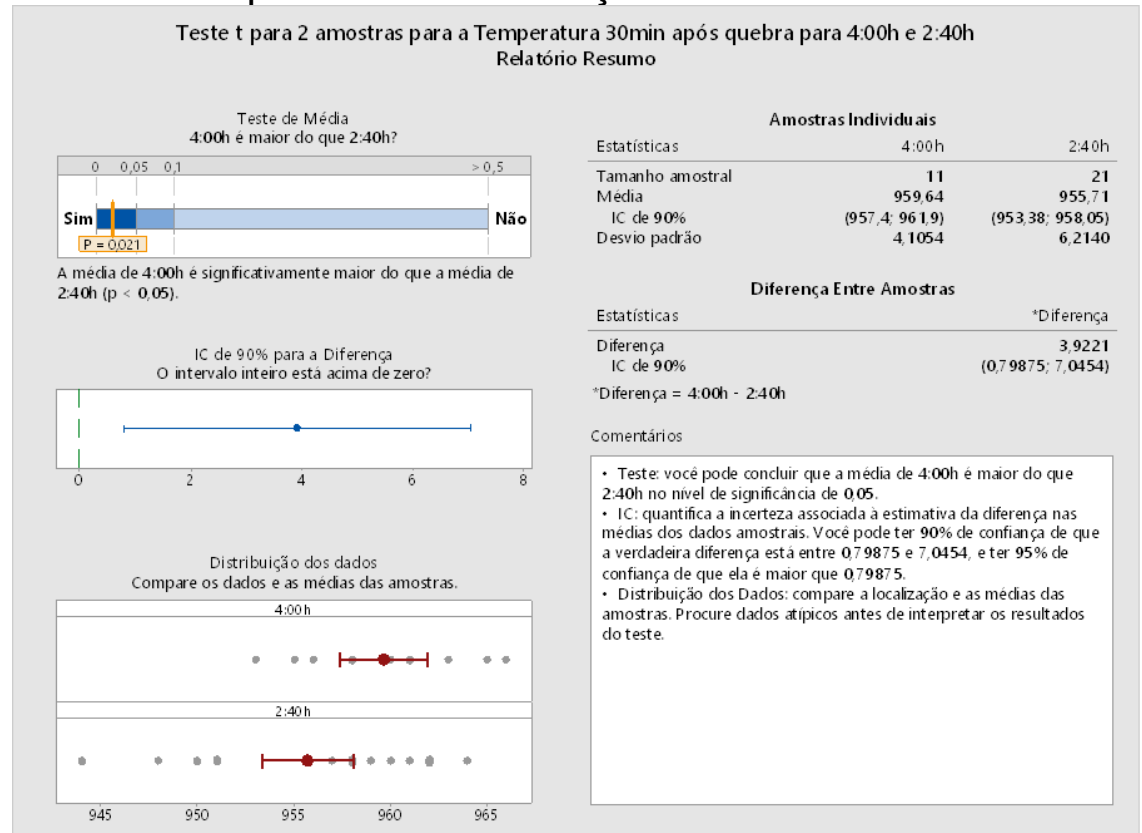

Figura 5 - Teste de Hipótese comparando a temperatura no tempo de 30 minutos após a quebra para os intervalos de 2:40 e 4:00 horas.

$\mathrm{Na}$ figura 5, foi avaliado o intervalo de tempo de 30 minutos, onde percebeu-se diferença estatística na temperatura entre as quebras de $3,9^{\circ} \mathrm{C}$, com a média em $959,6^{\circ} \mathrm{C}$ para a alimentação de $4: 00$ horas e $955,7^{\circ} \mathrm{C}$ para $2: 40$ horas. Com intervalos de trabalho entre 957,$4 ; 961,9^{\circ} \mathrm{C}$ e 953,$4 ; 958^{\circ} \mathrm{C}$ para $4: 00$ horas e $2: 40$ horas respectivamente. Apesar da temperatura média para 2:40 estar em um patamar mais elevado $955,7^{\circ} \mathrm{C}$, ainda se percebe uma grande quantidade das amostras operando em temperaturas abaixo de $954^{\circ} \mathrm{C}$, temperatura esta que favorece o processo de formação de lama.

Para os tempos seguintes não se realizou os testes de comparação pelo MINITAB devido ao comportamento de praticamente equiparação das médias de temperaturas apresentadas ao longo do tempo, onde além de aumentarem as mesmas se aproximam após 60 minutos da alimentação.

Ç

\subsection{Temperatura da crosta}

Para se ter uma maior precisão da temperatura na qual a alumina da crosta entra no banho eletrolítico para os diferentes tipos de quebra, foram feitas medidas da temperatura 5 minutos antes da quebra, em 6 cubas diferentes e em três pontos da crosta de acordo com a figura 6: parte inferior P3 (aproximadamente $5 \mathrm{~cm}$ da parte inicial da crosta), meio P2 (profundidade de aproximadamente $10 \mathrm{~cm}$ ) e parte superior P1 (4cm para a parte superior da crosta). 


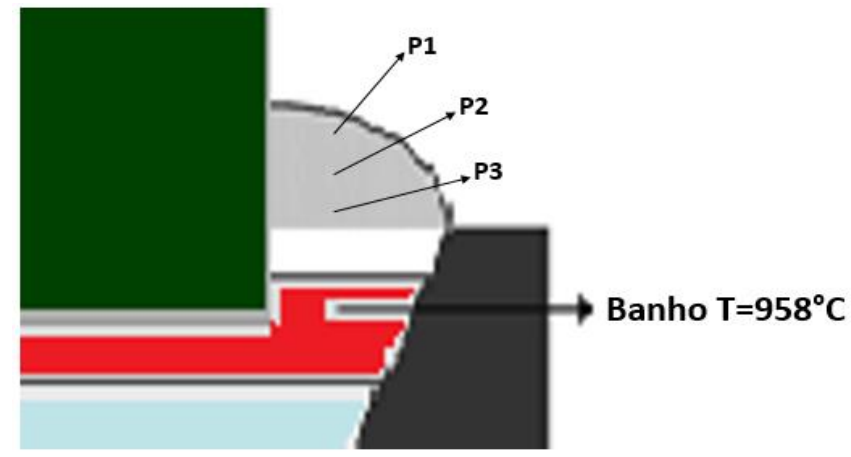

Figura 6 - Desenho esquemático dos pontos de medição da temperatura da crosta.

Os resultados encontrados foram plotados em um gráfico de intervalos que mostra uma pequena variação de $2^{\circ} \mathrm{C}$ na temperatura para a parte inferior em ambos regimes de alimentação, uma maior diferença para o meio e parte superior da crosta 66 e $72^{\circ} \mathrm{C}$ respectivamente, mas que pela amostragem não se pode dizer que as temperaturas são estatisticamente diferentes, mostrado pela figura 7 . Portanto baseado nessas temperaturas não podemos afirmar que a temperatura da crosta foi fator que causasse diferenciação na velocidade de dissolução para os diferentes regimes de alimentação. Outra observação importante é que pelo fato da temperatura da crosta ser semelhante, a mesma pode ter contribuído para que as curvas tanto da concentração de alumina no tempo quanto a velocidade de dissolução, tivessem o mesmo perfil.

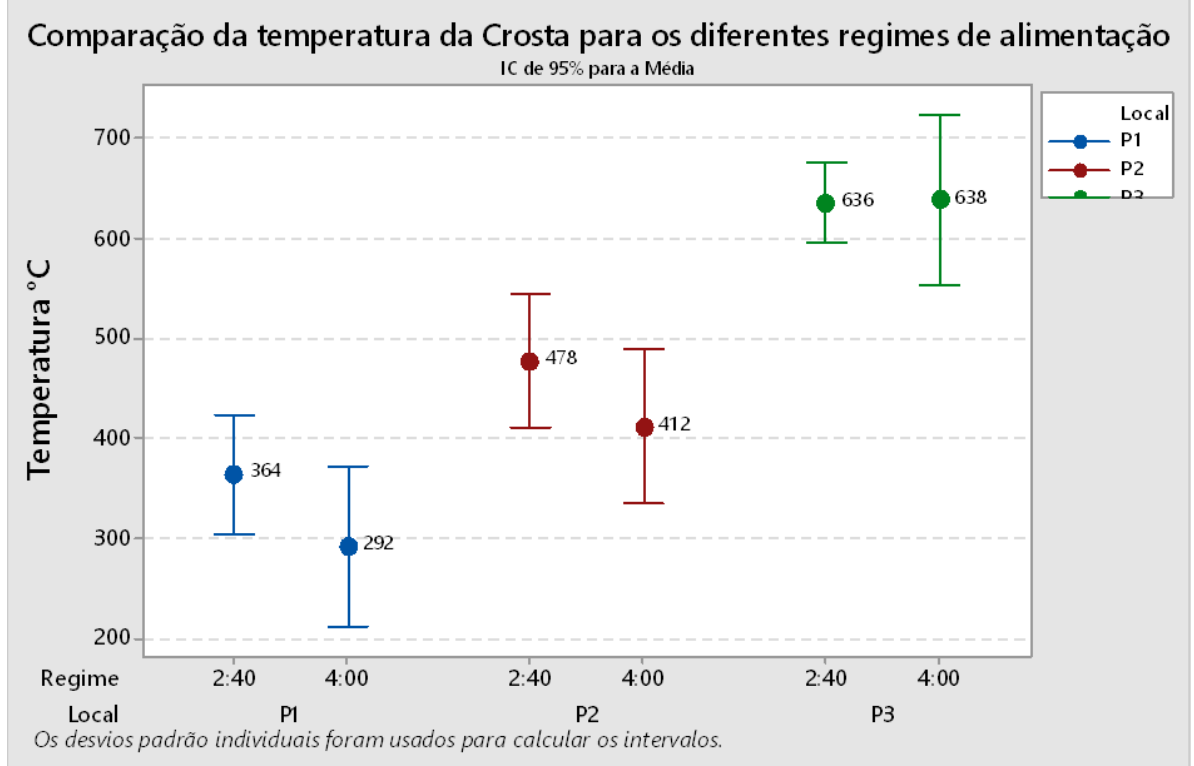

Figura 7 - Comparação da temperatura da crosta em diferentes pontos para os regimes de alimentação de 2:40 x 4:00 horas.

\subsection{Sondagem da cavidade das cubas}

A medição da cavidade é um importante fator a ser avaliado não para explicar a formação da lama, mas sim comprovar se existe um processo de formação de lama no fundo das cubas. Portanto foram medidas 9 cubas para o regime de 2:40 horas e 16 cubas para 4:00, com idade média de 30 meses e que operavam em condições normais de processo. Foram sondados três pontos diferentes das cubas, cabeceira A (1_Cab A), Meio (2_Meio) e a cabeceira B (3_Cab B). Os resultados obtidos estão mostrados no gráfico de intervalos na figura 8 .

As medidas de cavidade mostraram uma grande variação de deposição de lama entre as três regiões da cuba, onde a cabeceira $B$, principalmente para o regime de 
quebra de $2: 40$ possui uma perda média de 15,9 centímetros em relação a cabeceira A e de 13,4 centímetros em relação a cabeceira B dos fornos que trabalham em regime de 4:00. Esta perda de cavidade na cabeceira $B$ é atribuída a uma pior dissolução da alumina no banho ao longo do tempo para o regime de 2:40 horas, associada a uma movimentação do metal devido a configuração magnética do forno que arrasta a lama formada para o local.

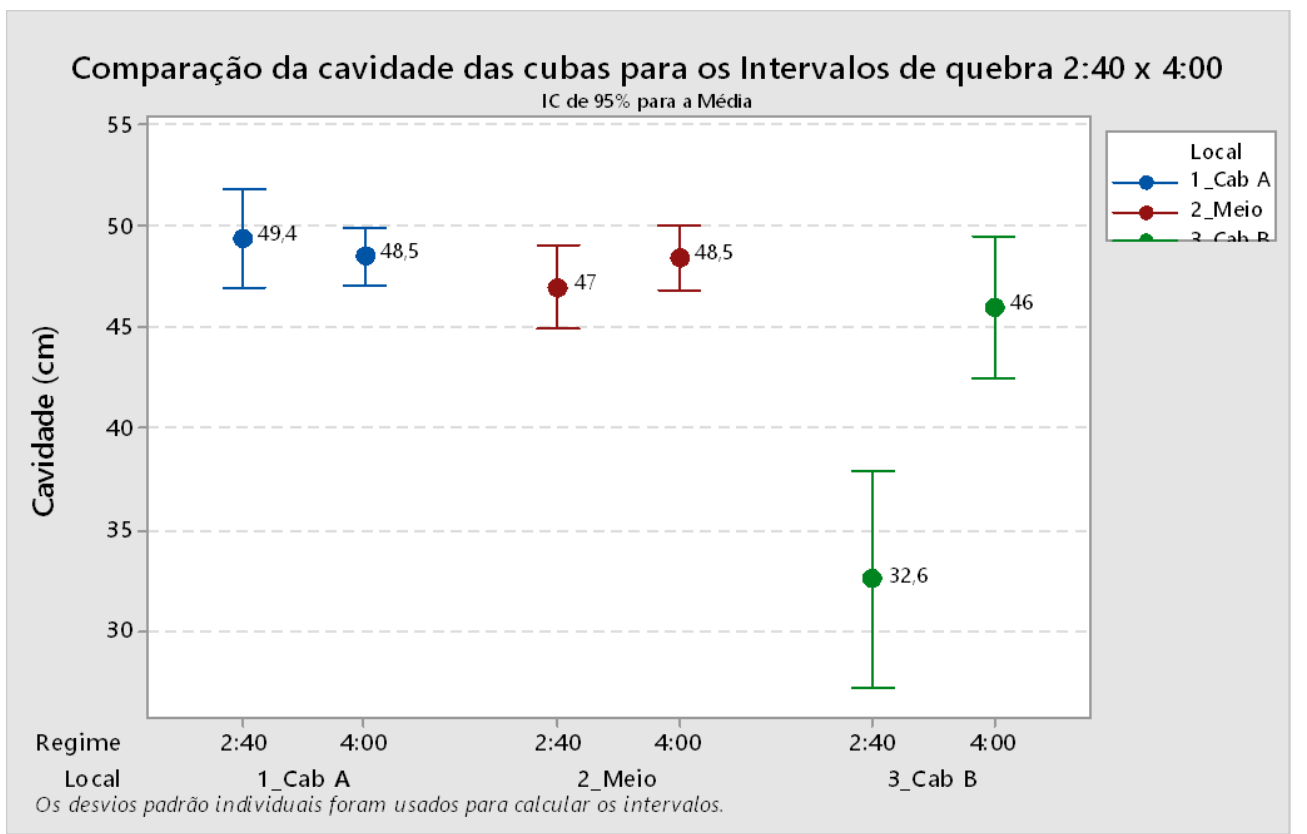

Figura 8- Sondagem comparativa da cavidade das cubas para os intervalos de 2:40 x 4:00 horas.

A perda de cavidade das cubas extremamente preocupante tendo em vista que a lama depositada e possível formação de cascão, poderão comprometer a passagem da corrente elétrica devido não homogeneidade na distribuição da corrente que além de comprometerem a movimentação magneto-hidrodinâmica do metal devido a correntes horizontais e concentrações de correntes localizadas, poderão causar problemas no anodo que consequentemente reduzirão a eficiência de corrente da cuba.

\section{Conclusões}

De acordo com os resultados, percebeu-se que o regime de 4:00 horas apresenta uma maior concentração de alumina no banho ao longo do tempo. Ocorrendo redução da concentração em todos os casos após a quebra (fato associado a amostragem do lado oposto a alimentação) e a subida logo em sequência para após 30 minutos iniciar a queda da concentração para o regime de alimentação de 2:40 horas e 60 minutos para 4:00 horas.

As medições de temperatura mostraram uma operação média para o regime de 4:00 horas de $2,69^{\circ} \mathrm{C}$ acima da $2: 40$ horas, que poderá induzir a uma perda de eficiência de corrente, mas as observações da temperatura nos intervalos de 15 e 30 minutos após alimentação, mostraram claramente que para os casos da alimentação de 2:40 horas existe uma grande quantidade de cubas apresentando temperatura significativamente baixas (abaixo de $954^{\circ} \mathrm{C}$ ) que contribuem para o processo de formação da lama e cascão.

As medições de temperatura da crosta, mostraram a alumina em uma temperatura não homogênea, com pontos variando entre 300 e $650^{\circ} \mathrm{C}$, que associada a grande 
quantidade adicionada contribuirá consequentemente para a aglomeração das partículas devido a intensa troca de calor banho/alumina, causando o processo de sedimentação de partículas e formação de lama.

As sondagens de fundo mostraram uma perda de cavidade na cabeceira B para as cubas com regime de 2:40, que deixam fortes evidencias que a formação da lama é fortemente influenciada pela baixa temperatura na qual o regime opera.

Devido os testes realizados ocorrerem em ambiente industrial com as cubas em operação normal, os resultados consequentemente são influenciados pelas variáveis de operação (calibração das balanças dos alimentadores, habilidade do operador, sistema de automação) e processo (química de banho, temperatura, massa de banho e massa de alumina adicionada) não possibilitando uma conclusão de qual regime de alimentação é o melhor. Portanto para se evitar a formação de lama no fundo das cubas, recomenda-se uma prática de processo e alimentação em batelada que: Consiga operar com temperaturas mínimas de no máximo $954^{\circ} \mathrm{C}$, trabalhar com o melhor controle de cargas possível de forma a alimentar a menor quantidade de alumina que se conseguir por cada adição, a existência de um volume adequado de banho que permita solubilização da alumina em grandes quantidades, além de operadores capacitados a realizarem a rotina operacional com excelência.

\section{REFERÊNCIAS}

1 WELTCH, B.J.;KUSCHEL, G.I. Crust and Alumina Powder Dissolution in Aluminium Smelting Electrolytes. JOM, 2007: 50-54.

2 DEWING, E. W. Loss of Current Efficiency in Aluminum Electrolysis Cells. Metallurgical and Materials Transactions B, April, 1991: 177-182.

3 VERSTREKEN, P. Superheat Sensor for Electrolytic Hall-Heroult Cells. Molten Slags. Fluxes and Salts. Conference, 1997.

4 SOLHEIM, A.; ROLSETH, S.; SKYBAKMOEN, E.; STOEN, L. Líquidus Temperature and Alumina Solubility in the System $\mathrm{Na}_{3} \mathrm{AlF}_{6}-\mathrm{AlF}_{3}-\mathrm{LiF}-\mathrm{CaF}_{2}-\mathrm{MgF}_{2}$. Light Metals, 1995: 451-460.

5 KUSCHEL, G.I.; WELTH B.J. Further Studies of Alumina Dissolution under Conditions Similar to Cell Operation. Light Metals, 1994: 299-305.

6 HAUPIN, W.; JARRETT, N.; HUDSON, L.K.; COCHRAN, N. Production of aluminium and alumina. 1983.

7 THONSTAD, J.; NORDMO, F.; PAULSEN, J. B. Dissolution of Alumina in Molten Cryolite. Metallurgical Transactions, 1972: 403.

8 LUBYOVÁ, Z.; FELLNER, P.; GABCOVÁ, J. Solubility and Rate of Dissolution of Alumina in Cryolite-Based Alf3-Rich Melts with Additions of LiF, CaF2, and MgF2. Institute of Inorganic Chemistry, Slovak Academy of Sciences, September 1982.

9 GRJOTHEIM, K.; KVANDE, H. Understanding the Hall-Héroult Process for Production of Aluminium. Aluminium Verlag. Dusseldorf. Oslo. September, 1986.

10 BARBER, M. Manual de Treinamento de Química de Banho. Technical Information Center. Alcan. Ouro Preto, 2002.

11 SILVA, G. Manual de Metalurgia do Alumínio. Treinamento Operacional. Votorantim CBA. Alumínio, 1997.

12 KELLER, R. Alumina Dissolution and Sludge Formation. Light Metals, 1984, p. 513-18.

13 WANG, X. Alumina Dissolution in Aluminum Smelting Electrolyte. Light Metals. 2009: 383-388.

14 CASTRO, RICARDO H. R. Estudo da Influência de Aditivos na Transformação de Fase Gama-Alfa da Alumina. Escola Politécnica da Universidade de São Paulo, São Paulo, 2005.

15 TOWNSEND, D. W. \& BOXALL, L. G. Crushing Behavior of Smelter Aluminas. Martin Marietta Laboratories. Light Metals, 1984: 649-664. 